\title{
Aplicación del costeo basado en actividades en las Universidades
}

\begin{abstract}
RESUMEN
El presente artículo muestra cómo el costeo basado en actividades puede ser aplicado en la docencia universitaria, y cómo esta herramienta de gestión puede ayudar a una mejor administración de recursos.

Palabras claves: Gestión universitaria, Costeo Basado en Actividades, educación superior.

Applying ACtivity Based Costing IN UNIVERSITIES

ABSTRACT

This article shows how the activity based costing can be applied to higher education, and how this management tool can help to improve the resources management.
\end{abstract}

Key words: University management, Activity based costing, Higher education

\section{INTRODUCCIÓN}

El tema del costeo basado en actividades ha sido tratado extensamente por autores como Barfield et al (2004), Bellido (2003), Brewer et al (2002), Hilton et al (2003), y Horngren et al (2006), Asimismo, la Revista Industrial Data ha publicado artículos de Carrión (2005), y Vergiú (2001 y 2005) que define el concepto de costeo basado en actividades y lo ilustran con ejemplos aplicados a la industria.

Aunque el enfoque inicial del costeo basado en actividades fue en la actividad empresarial, posteriormente los administradores universitarios consideraron conveniente utilizar esta herramienta de gestión, y es así como hace diez años la prestigiosa institución Scottish Higher Education Funding Council for England publicó el documento Management Information for Decision Making: Costing Guidelines for Higher Education Institutions (1997), en dicho documento el profesor David Westbury estableció que "un adecuado costeo para la toma de decisiones en universidades es vital, en especial cuando existen restricciones de presupuesto", si se compara esta expresión con nuestras universidades públicas, podremos apreciar que nuestra realidad no se escapa de dicha condición.

Asimismo, en 1996 los profesores Kober, Brown, Izan, y Robinson de la University of Western Australia condujeron el proyecto de investigación "Costing Human Resources Services in an Australian University: An Application of Activity Based Costing (1996), ilustran cómo el sistema de costeo basado en actividades puede ser utilizado en las universidades.

Por otro lado, los doctores Michael H, Granof, David E, Platt, y Igor Vaysaman (2000) consideran que "los administradores de las universidades conocen el valor de todo, pero no conocen el costo de nada", y en tal sentido desarrollaron un estudio que muestra como el Costeo Basado en Actividades debería aplicarse en la actividad académica.

Estudios más recientes realizados por las profesoras Teresa García Valderrama y Rosario del Río Sánchez de la Universidad de Cádiz en España publicaron el artículo Desarrollo e Implementación de un Modelo de Costeo para Universidades (2006), que analiza las ventajas y desventajas de utilizar el sistema de costeo basados en actividades en universidades españolas.

\section{PRESENTACIÓN DEL MODELO}

El documento Costing Guidelines for Higher Education Institutions (1997) establece que el proceso de costeo basado en actividades para

(1) Magíster en Ciencias. Profesor del Departamento de Producción y Gestión Industrial, UNMSM E-mail: jdelcarpiog@unmsm.edu.pe 
Cuadro 1. Modelo de Departamento Académico

\begin{tabular}{|l|l|l|}
\hline \multicolumn{1}{|c|}{ Recursos } & \multicolumn{1}{c|}{ Actividades } & \multicolumn{1}{c|}{ Productos } \\
\hline Personal docente & Docencia & Cursos \\
\hline Asistentes de investigación & Investigación & Publicaciones \\
\hline Personal administrativo & Actividad académica & Proyectos \\
\hline Personal técnico & Consultoría & Reportes \\
\hline Suministros & Gestión universitaria & Otros \\
\hline Servicios & & \\
\hline Equipos & & \\
\hline Edificios & & \\
\hline
\end{tabular}

Fuente: Costing Guidelines for Higher Education Institutions (1997)

universidades es como sigue:

- Identificar los costos de los recursos (recursos humanos, consumibles, equipos, etc.)

- Identificar los productos (cursos, reportes de investigación, trabajos de consultoría, y otros)

- Identificar las actividades (docencia, investigación, procesos de admisión, servicios de biblioteca, servicios académicos)

- Asignar los costos de los recursos a las actividades

- Relacionar las actividades con los productos usando inductores de costos (personal docente, estudiantes, espacio, etc.)

- Analizar y reportar los resultados

Por otro lado el documento anteriormente mencionado, establece el modelo para un departamento académico, el cual se presenta en el cuadro 1.

\section{APLICACIÓN DEL MODELO EN LA FACULTAD DE INGENIERÍA INDUSTRIAL DE LA UNMSM}

Para aplicar el modelo de costos basados en actividades en el Laboratorio de Informática de la Facultad de Ingeniería Industrial, ubicado en el cuarto piso del Pabellón de Aulas, se procederá con cada uno de los pasos sugeridos:

\section{Paso 1: Identificar los costos de los recursos}

En el Laboratorio de Computación de la Facultad de Ingeniería Industrial, se ofrecen clases prácticas de los cursos de pre y post grado, En primer lugar se tiene los costos directos, constituidos por la actividad de docencia ejercida por los docentes, se esta considerando que el costo de tasa horaria de un docente de pregrado es de S/. 20, en tanto, que para el docente de postgrado la tasa horaria es de S/. 50 .

El cuadro 2 muestra la relación de equipos que se utilizan en el laboratorio antes mencionado, los costos de los equipos son referenciales, y se ha considerado un periodo contable de recuperación de la inversión de cuatro años, La depreciación y amortización se han estimado para el periodo de un mes.

El cuadro 3 presenta la relación de recursos humanos, considerados como costos indirectos para el periodo de un mes.

El cuadro 4 muestra el resumen mensual de los costos indirectos que se incurren en equipos, recursos humanos, servicios, gestión de la Facultad de Ingeniería Industrial, y uso del espacio físico.

\section{Paso 2: Identificar los productos}

El Cuadro 5 muestra la relación de los cursos que se ofrecen en el mencionado laboratorio, señalando además el número de horas mensuales y la cantidad de alumnos registrados en cada sección.

\section{Paso 3: Identificar las actividades}

Siendo el Laboratorio de Informática de la Facultad de Ingeniería Industrial de la UNMSM orientado básicamente a la docencia, se ha considerado que los inductores de costos de actividad docencia sean la cantidad de horas académicas y el número de alumnos que hacen uso del laboratorio. Para el semestre 2007-I, las horas académicas ascendieron a 240 horas, y la cantidad de alumnos matriculados fue de 426 .

Paso 4: Asignar los costos de los recursos a las actividades

En el cuadro 6 se presenta el cálculo de las tasas de asignación de las tasas de costos indirectos ha sido elaborado considerando la información que se muestra en el Cuadro No. 4 Relación de costos indirectos del Laboratorio, y los inductores de costos mencionados en el paso 3

\begin{tabular}{|c|c|c|c|c|}
\hline \multicolumn{5}{|c|}{$\begin{array}{c}\text { Cuadro 2. Relación de equipos en el laboratorio } \\
\text { de computación de la FII UNMSM }\end{array}$} \\
\hline Descripción & Cant. & \begin{tabular}{|c|} 
Costo \\
Unitario S/.
\end{tabular} & \begin{tabular}{|c|} 
Valor \\
total S/.
\end{tabular} & $\begin{array}{c}\text { Depreciación } \\
\text { Amortización S/. }\end{array}$ \\
\hline Servidor HP Proliant ML 110G4 & 1 & 5000,0 & 5000,0 & 104,17 \\
\hline $\begin{array}{l}\text { Computadora Core } 2 \text { Duo 1,86 Ghz, } \\
\text { 1Gb RAM, 160Gb HDD }\end{array}$ & 30 & 2700,0 & 81000,0 & 1687,50 \\
\hline $\begin{array}{l}\text { Licencias de Software Windows } \\
\text { XP Profesional SP2 }\end{array}$ & 31 & 600,0 & 18600,0 & 387,50 \\
\hline $\begin{array}{l}\text { Licencias de Software Aplicativo } \\
\text { MS-Office } 2003 \text { Prof, }\end{array}$ & 31 & 1300,0 & 40300,0 & 839,58 \\
\hline Mobiliario & 12 & 500,0 & 6000,0 & 125,00 \\
\hline Cableado Categoría 6 Panduit & 1 & 4000,0 & 4000,0 & 83,33 \\
\hline $\begin{array}{l}\text { Equipo de Comunicación Switch } \\
\text { Cisco Catalisys } 296024 \mathrm{P}\end{array}$ & 1 & 3500,0 & 3500,0 & 72,92 \\
\hline Estabilizadores & 31 & 60,0 & 1860,0 & 38,75 \\
\hline Proyector multimedia Panasonic PT & 1 & 6000,0 & 6000,0 & 125,00 \\
\hline Repuestos & 10 & 500,0 & 5000,0 & 104,17 \\
\hline Total & & & & 3567,92 \\
\hline
\end{tabular}

Fuente: Oficina de Informática y Estadística de la Facultad de Ingeniería Industrial Julio 2006 
Cuadro 3. Relación de recursos humanos

\begin{tabular}{|l|r|r|r|}
\hline \multicolumn{1}{|c|}{$\begin{array}{c}\text { Recursos } \\
\text { Humanos }\end{array}$} & $\begin{array}{c}\text { Tarifa } \\
\text { Cantidad } \\
\text { (Horas) }\end{array}$ & $\begin{array}{c}\text { Horaria } \\
\text { S/. }\end{array}$ & $\begin{array}{c}\text { Valor } \\
\text { Total S/. }\end{array}$ \\
\hline Personal soporte & 280 & 5,0 & 1400,00 \\
\hline Ayudantes de cátedra & 280 & 3,0 & 840,00 \\
\hline Limpieza & 48 & 2,0 & 96,00 \\
\hline Total & & & $\mathbf{2 3 3 6 , 0 0}$ \\
\hline
\end{tabular}

Fuente: Dirección Administrativa FII UNMSM Julio 2006

Paso 5: Relacionar las actividades con los productos usando inductores de costos

En el Cuadro 7 se muestra los costos directos y costos indirectos de cada uno de los cursos. Así mismo, se ha calculado el costo unitario por alumno de cada uno de los cursos ofrecidos en el laboratorio en el semestre 2007-I.

Paso 6: Analizar y reportar los resultados

Con la información obtenida ya se esta en condiciones de responder a preguntas como:

Cuadro 5. Relación de cursos que se ofrecieron en el Laboratorio - Semestre 2007-I

\begin{tabular}{|l|r|r|}
\hline \multicolumn{1}{|c|}{ Productos } & $\begin{array}{r}\text { Cantidad } \\
\text { de Horas } \\
\text { Mensual }\end{array}$ & $\begin{array}{c}\text { Cantidad } \\
\text { de } \\
\text { Alumnos }\end{array}$ \\
\hline Algoritmos y Programación Sección 1 & 16 & 22 \\
\hline Algoritmos y Programación Sección 2 & 16 & 22 \\
\hline Algoritmos y Programación Sección 3 & 16 & 23 \\
\hline Algoritmos y Programación Sección 4 & 16 & 23 \\
\hline Métodos Computacionales Sección 1 & 16 & 30 \\
\hline Métodos Computacionales Sección 2 & 16 & 30 \\
\hline Métodos Computacionales Sección 3 & 16 & 30 \\
\hline Investigación Operativa II Sección 1 & 8 & 24 \\
\hline Investigación Operativa II Sección 2 & 8 & 24 \\
\hline Simulación y Modelación & 8 & 30 \\
\hline Estadística Industrial & 16 & 30 \\
\hline Estadística y Probabilidades & 16 & 30 \\
\hline Ingeniería de Software & 8 & 30 \\
\hline Informática & 16 & 30 \\
\hline Curso Postgrado Sección 1 & 12 & 12 \\
\hline Curso Postgrado Sección 2 & 12 & 12 \\
\hline Curso Postgrado Sección 3 & 12 & 12 \\
\hline Curso Postgrado Sección 4 & 12 & 12 \\
\hline Total & $\mathbf{2 4 0}$ & $\mathbf{4 2 6}$ \\
\hline
\end{tabular}

Fuente: Jefatura de Laboratorios de Computación FII UNMSM
Cuadro 4. Relación de costos indirectos del Laboratorio

\begin{tabular}{|l|r|}
\hline \multicolumn{1}{|c|}{ Costos Indirectos } & \multicolumn{1}{c|}{$\begin{array}{c}\text { Valor } \\
\text { Total S/. }\end{array}$} \\
\hline Equipos & 3567,92 \\
\hline Recursos Humanos & 2336,00 \\
\hline Servicios & 240,00 \\
\hline Gastos Gestión de la Facultad & 2400,00 \\
\hline Espacio físico & 1200,00 \\
\hline Total & $\mathbf{9 7 4 3 , 9 2}$ \\
\hline
\end{tabular}

Fuente: Elaboración propia,

- ¿Se debe convalidar las notas de laboratorio?. Considerando los resultados las notas de laboratorio deben convalidarse.

- ¿La primera repitencia en un curso que hace uso de laboratorio debe ser cero?. Los cálculos muestran que el costo no debería ser cero.

- ¿Debe tener costo el uso de laboratorio de cómputo para poder reponerlo tecnológicamente? De no tener costo el laboratorio de computación de la Facultad de Ingeniería Industrial de la UNMSM enfrentará la posibilidad de desfasarse tecnológicamente.

\section{CONCLUSIONES}

El costeo basado en actividades puede ser aplicado a las universidades, asimismo, la información obtenida mediante la aplicación de esta herramienta ayudaría a tomar mejores decisiones en estas organizaciones.

Por otro lado, el costeo basado en actividades ayuda a realizar un mejor uso de los recursos en las universidades, más aún si son públicas.

Cuadro 6. Cálculo de las tasas de asignación de las tasas de costos indirectos.

\begin{tabular}{|l|r|r|r|r|}
\hline \multicolumn{1}{|c|}{ Recursos } & Valor Total S/. & $\begin{array}{c}\text { Cantidad } \\
\text { de Horas }\end{array}$ & $\begin{array}{c}\text { Cantidad de } \\
\text { alumnos }\end{array}$ & $\begin{array}{r}\text { Inductor } \\
\text { de costo }\end{array}$ \\
\hline Equipos & 3567,92 & 240 & & 14,87 \\
\hline Recursos Humanos & 2336,00 & 240 & & 9,73 \\
\hline Servicios & 240,00 & & 426 & 0,56 \\
\hline $\begin{array}{l}\text { Gastos Gestión de } \\
\text { la Facultad }\end{array}$ & 2400,00 & & 426 & 5,63 \\
\hline Espacio físico & 1200,00 & & 426 & 2,82 \\
\hline Total S/. & 9743,92 & & & \\
\hline
\end{tabular}

Fuente: Elaboración propia. 


\begin{tabular}{|c|c|c|c|c|c|c|c|c|c|c|}
\hline & $\begin{array}{l}\text { Cantidad } \\
\text { de Horas }\end{array}$ & $\begin{array}{c}\text { Cantidad } \\
\text { de } \\
\text { Alumnos }\end{array}$ & $\begin{array}{c}\text { Costo } \\
\text { Directo } \\
\text { Docente }\end{array}$ & Equipos & $\begin{array}{l}\text { Recursos } \\
\text { Humanos }\end{array}$ & Servicios & $\begin{array}{c}\text { Gastos de } \\
\text { Gestión }\end{array}$ & $\begin{array}{c}\text { Espacio } \\
\text { Físico }\end{array}$ & $\begin{array}{l}\text { Costo } \\
\text { Total }\end{array}$ & $\begin{array}{c}\text { Costo por } \\
\text { alumno }\end{array}$ \\
\hline Algoritmo Sección 1 & 16 & 22 & 320,00 & 237,86 & 155,73 & 12,39 & 123,94 & 61,97 & 911,90 & 41,45 \\
\hline Algoritmo Sección 2 & 16 & 22 & 320,00 & 237,86 & 155,73 & 12,39 & 123,94 & 61,97 & 911,90 & 41,45 \\
\hline Algoritmo Sección 3 & 16 & 23 & 320,00 & 237,86 & 155,73 & 12,96 & 129,58 & 64,79 & 920,92 & 40,04 \\
\hline Algoritmo Sección 4 & 16 & 23 & 320,00 & 237,86 & 155,73 & 12,96 & 129,58 & 64,79 & 920,92 & 40,04 \\
\hline Métodos Computacionales Sección 1 & 16 & 30 & 320,00 & 237,86 & 155,73 & 16,90 & 169,01 & 84,51 & 984,02 & 32,80 \\
\hline Métodos Computacionales Sección 2 & 16 & 30 & 320,00 & 237,86 & 155,73 & 16,90 & 169,01 & 84,51 & 984,02 & 32,80 \\
\hline Métodos Computacionales Sección 3 & 16 & 30 & 320,00 & 237,86 & 155,73 & 16,90 & 169,01 & 84,51 & 984,02 & 32,80 \\
\hline Investigación Operativa II Sección 1 & 8 & 24 & 160,00 & 118,93 & 77,87 & 13,52 & 135,21 & 67,61 & 573,14 & 23,88 \\
\hline Investigación Operativa II Sección 2 & 8 & 24 & 160,00 & 118,93 & 77,87 & 13,52 & 135,21 & 67,61 & 573,14 & 23,88 \\
\hline Simulación & 8 & 30 & 160,00 & 118,93 & 77,87 & 16,90 & 169,01 & 84,51 & 627,22 & 20,91 \\
\hline Estadística Industrial & 16 & 30 & 320,00 & 237,86 & 155,73 & 16,90 & 169,01 & 84,51 & 984,02 & 32,80 \\
\hline Estadística y Probabilidades & 16 & 30 & 320,00 & 237,86 & 155,73 & 16,90 & 169,01 & 84,51 & 984,02 & 32,80 \\
\hline Ingeniería de Software & 8 & 30 & 160,00 & 118,93 & 77,87 & 16,90 & 169,01 & 84,51 & 627,22 & 20,91 \\
\hline Informática & 16 & 30 & 320,00 & 237,86 & 155,73 & 16,90 & 169,01 & 84,51 & 984,02 & 32,80 \\
\hline Curso Postgrado Sección 1 & 12 & 12 & 600,00 & 178,40 & 116,80 & 6,76 & 67,61 & 33,80 & 1003,36 & 83,61 \\
\hline Curso Postgrado Sección 2 & 12 & 12 & 600,00 & 178,40 & 116,80 & 6,76 & 67,61 & 33,80 & 1003,36 & 83,61 \\
\hline Curso Postgrado Sección 3 & 12 & 12 & 600,00 & 178,40 & 116,80 & 6,76 & 67,61 & 33,80 & 1003,36 & 83,61 \\
\hline Curso Postgrado Sección 4 & 12 & 12 & 600,00 & 178,40 & 116,80 & 6,76 & 67,61 & 33,80 & 1003,36 & 83,61 \\
\hline \begin{tabular}{|c|} 
Total \\
\end{tabular} & & & 6240,00 & 3567,92 & 2336,00 & 240,00 & 2400,00 & 1200,001 & 15983,92 & \\
\hline
\end{tabular}

Fuente: Elaboración propia. Las cifras de los costos están expresadas en Nuevos Soles.

\section{RECOMENDACIONES}

Continuar aplicando el costo basado en actividades a otras áreas de las actividades académicas y administrativas de la universidad. Así como ejecutar estudios comparativos que permitan aplicar el benchmarking, a fin de hacer más eficiente la gestión académica y administrativa en la Facultad de Ingeniería Industrial.

\section{REFERENCIAS BIBLIOGRÁFICAS}

1. Barfield, J,; Raiborn, C,; Kinney, M, (2004), Contabilidad de Costos, Tradiciones e Innovaciones, Quinta Edición, Thomson.

2. Bellido, P, (2003), ABC Activity Based Costing/ Costeo Basado en Actividades, Instituto de Investigación EI Pacifico E,I,R,L.

3. Brewer, P; Garrison, R,; Norreen, E, (2002), Introduction to Managerial Accounting, Second Edition, Mc Graw-Hill Irwin.

4. Carrión, J, (2205), Pautas básicas para una implantación exitosa del costeo basado en actividades, Industrial Data, Vol, 8, No, 1: 47-52.

5. Garcia, T,; Del Rio, R, (2006) Development and Implementation of a University Costing Model, Public Money and Management, September 2006: 251-255.
6. Granof, M; Platt, D,; Vaysman, I, (2000) Using Activity Based Costing to Manage More Effectively, Department of Accounting, College of Business Administration, University of Texas at Austin, January 2000

7. Hilton, R,; Maher, M; Selto, F, (2003), Cost Management: strategies for business decisions, Second edition, Mc Graw-Hill Higher Education.

8. Horngren, Ch,; Sundem, G,; Stratton, W, (2006), Contabilidad Administrativa, Décimo Tercera Edición, Pearson Educación.

9. Scottish Higher Education Funding Council, Higher Education Funding Council for England (1997), Higher Education Funding Council for Wales, July 1997

http://www,sfc,ac,uk/publications/pubs_other_ shefcarchive/costing/contents, htm

Management Information for Decision-Making: Costing Guidelines for Higher Education Institutions,

(Página visitada el día 2 de agosto 2007)

10. Vergiu, J, (2001) Evaluación de la Gestión Empresarial; Costos por Actividades, Industrial Data, Vol, 4, No, 1: 34-35.

11. Vergiú, J, (2005) Rentabilidad por producto mediante el costeo basado en actividades: Caso del sector industrial, Industrial Data, Vol, 8, No, 1: 42-46. 